\title{
Automation Not Automatically Good in Mobile Social Applications
}

\author{
Sami Vihavainen, Antti Oulasvirta, Risto Sarvas, Helsinki Institute for Information Technology HIIT / \\ Helsinki University of Technology TKK
}

\begin{abstract}
Social interaction is increasingly computer mediated. Part of the mediated interaction is being automated by the technology used, especially in mobile phone technology. Human factors research has shown that automation is a mixed blessing. It changes the role of the human in the loop with effects on understanding, control, skill, vigilance, and ultimately trust and usefulness. The question we want to raise is: What are the implications of increasing automation in mobile social applications?

Jaiku, a mobile awareness service, automates disclosure and diffusion of location metadata, and ZoneTag, a photo-uploading program, automates and suggests metadata associated with images. We discuss alternative models of automation in these systems and present a study of three user groups in Finland and California using Jaiku and ZoneTag. The results reveal issues related to control, understanding, emergent practices, and privacy. We discuss the potentials and limitations of automated solutions in this context and discuss about the need for further research on automation in mobile social applications.
\end{abstract}

\section{INTRODUCTION}

$\mathrm{H}$ UMAN factors research has shown that automation is a mixed blessing. It changes the role of the human in the loop with effects on understanding, control, skill, vigilance, and ultimately trust and usefulness [3]. But what are the implications of introducing automation into mobile social media? Which models of automation are acceptable? How do they affect interaction and use?

We consider this an interesting topic for the MobiQuitous community for two reasons: First, the reason that the "process" that is being "controlled" here is social by nature, not safety- or performance-related. The implications of automation to computer-mediated social interaction have not been systematically addressed. Second, we see automated solutions increasingly in this category of applications. The raison d'etre for adding automation in these applications is productivity: a mobile user does not have the time and resources to manually post and update this information.

This study was part of the Finnish Funding Agency for Technology and Innovation (TEKES) project Täky. Antti Oulasvirta was supported by the Academy of Finland project Amoveo.

S. Vihavainen is with Helsinki Institute for Information Technology HIIT / Helsinki University of Technology TKK P.O. Box 9800, FIN-02015 HUT, FINLAND (phone: +358 44348 2517, e-mail: sami.vihavainen (at) hiit.fi).

A. Oulasvirta is with Helsinki Institute for Information Technology HIIT / Helsinki University of Technology TKK, P.O. Box 9800, FIN-02015 HUT, FINLAND (e-mail: antti.oulasvirta (at) hiit.fi).

R. Sarvas is with Helsinki Institute for Information Technology HIIT / Helsinki University of Technology TKK, P.O. Box 9800, FIN-02015 HUT, FINLAND (e-mail: risto.sarvas (at) hiit.fi).
Instead of general aspects of user experience of the user interface, we focus on automated features that in a way "exist" beyond the UI in the system that collects and propagates location information to other users. Our goal is to shed light on three questions, the first two we believe are unique to mobile social applications. The third has been studied in human factors, but not in this application context:

1. Use of automation in mobile social applications?

2. User response to automation?

3. Users' understanding of the logic of automation?

By studying three user groups and two systems our goal is to gain a more general view to the subject, instead of pooling results from an individual system. Interviews and log analysis were used as the main methods of data collection. We base our argumentation on comparisons between the systems and the groups. We investigate Jaiku and ZoneTag as applications that partly automate the social interaction processes.

\section{II.AN OVERVIEW OF THE TWO APPLICATIONS}

Jaiku (jaiku.com) is is a Nokia S60-based mobile social application built on the ContextPhone platform [2]. It allows a group to share textual status updates that are associated with automatic location information which shows a label for the user's GSM cell ID. By location information we mean the information displayed in the user interface right after the status updates (e.g. in Fig. 1, for "Petteri Koponen" the location information is "London").

ZoneTag is a mobile social application that allows rapid publication of media on the phone to Internet. With ZoneTag, users can upload photos from a cameraphone to the Flickr Web service. ZoneTag can automatically supply location metadata to a photo using GSM cell ID data. Moreover, it offers an interface to assign tags to a photo, either by typing them or choosing from a list suggested by the system. [1]

Concerning the automated processes, in Jaiku we concentrate on user's current location information sharing and in ZoneTag on tags associated with images. In Jaiku, the process of choosing, assigning and disclosing the location label is initiated whenever the phone's cell tower changes. Thus, the user has practically no control over the initiation. In ZoneTag, the processes of choosing, assigning and disclosing the location tag and the list of suggested tags are initiated when the user has taken a photograph. Then the application prompts the user "Upload to Flickr?" and she can choose yes or no. Thus, the process is under the user's control. 


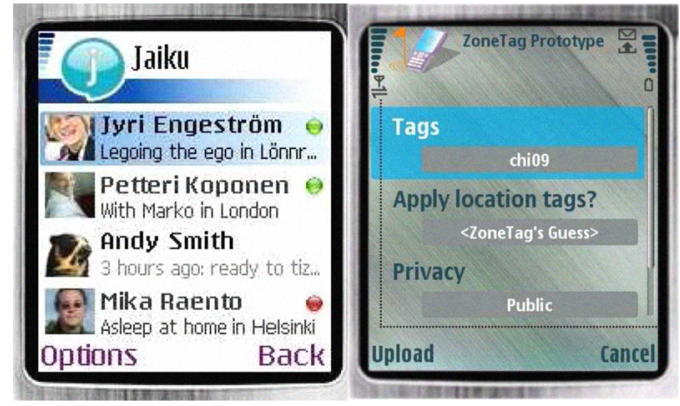

Fig 1. Left: Jaiku shows contacts' presence lines and real-time location cues. Right: ZoneTag's automation suggests tags to be added to photos.

\section{FIELD TRIALS}

Three groups (The Students, The Birders, The Hipsters) were recruited in Finland and California, and they used the two systems, each for two months, first the other then the other. A mix of quantitative (content, logs) and qualitative (interviews) methods was used in order to gauge both interaction on the phone and subjective views on automation.

\section{RESULTS}

The results showed how the automation related questions are essential factors affecting user experience of location based systems, and should be taken seriously when designing mobile social applications. The users had problems with all aspects of automation in both systems. In Jaiku, they did not always understand the geographic area covered by GSM cell IDs, the timeliness of the data when other users were off-line, or the purpose of real-time location disclosure. Only one group found real benefit from Jaiku-the Students ( 5 men, 5 women, aged 18-20) who used it for coordination and ad hoc encounters. In these pursuits, the automated location information was useful but often insufficient. The two other groups exhibited indifference and withdrawal toward Jaiku, but their reasons were different. Although the common activity of The Birders ( 7 men, 1 woman, aged 18-64), an interest group of birdwatchers, was spatial by nature, they did not have simultaneous collective efforts, and they did not know each other outside the interest groups, with the result that the automatic information was simply uninteresting and had not to be controlled. Automation was a non-issue to them. The Hipsters (4 men, 4 women, aged 28-32) were initially worried about privacy issues and generally felt they do not need such a channel as they already interact with each other enough. They rarely logged in and used it mainly to check others' availability.

The ZoneTag users did notice the existence of automation, but mostly neglected the suggested tags and location tag features. Automation was a non-issue unless it failed somehow. For example, one group struggled with cellID inaccuracy and had to manually "repair" those by commenting in Flickr. In another group, we found a user wanting to hide from others the place in which a picture was taken. Most of the time, however, the users did not really need to understand the logic of automation, because they did not care about the tags.

In reference to the three questions we set in the beginning, our observations can be summarized as the following set of claims about automation in mobile social applications.

Use of automation in mobile social applications:

1. Automation can threaten accountability of actions. When automated data is mixed with manual data in the UI, the danger is that the communicative functions of tags and manual location labels are lost

2. The effects of automation are accentuated in services where mediated interaction is more rapid and immediate.

\section{User response to automation:}

3. The negative effects of automation at the level of the group include neglect in the face of useless automation, and withdrawal in the face of a too threateningly strong model of automation.

4. Privacy concerns are felt but do not necessary actualize due to the measures the users can take both inside and outside the system.

5. The nature of shared activities, and the usefulness of the automated information therein, shapes whether automation will be useful, useless, or annoying. The level of automation, and its content, should be chosen according to what is known about these activities.

\section{Users' understanding of the logic of automation:}

6. The negative effects of automation on the individual reflect the classic findings of human factors. Particularly, ignorance of automation, misunderstanding of its operating logic, post hoc "repairs," repetitive behavior (resending location labels to ensure they appear), and eventually shutting down of the system can emerge.

\section{V.FURTHER RESEARCH}

We see automation in this ubiquitous world as an important and challenging field to continue our work. Interaction with increasingly smart mobile phones brings up questions like, what tasks should be automated and what left to human. Also a bigger question is how intelligent mobile applications should be designed so that they act in the interest of the user?

\section{ACKNOWLEDGEMENT}

We thank our collaborators Mor Naaman and Rahul Nair from Yahoo! for providing the ZoneTag data and Mika Raento from Jaiku for providing the Jaiku data.

\section{REFERENCES}

[1] Ahern, S., King, S., Naaman, M., Nair, R. and Yang, J.H.I. ZoneTag: Rich, Community-Supported Context-Aware Media Capture and Annotation. Proc. CHI 2007, ACM Press (2007).

[2] Raento, M., Oulasvirta., A., Petit, R. and Toivonen, H. ContextPhone: A prototyping platform for contextaware mobile applications. IEEE Pervasive Computing 4, 2 (2005), 51-59.

[3] Sheridan, T., Parasuraman, R. Human-Automation Interaction, Review of Human Factors and Ergonomics, 1 (2006), 89-129. 